\title{
Wafer-bonded single-crystal silicon slot waveguides and ring resonators
}

\author{
Ryan M. Briggs, ${ }^{\text {a) }}$ Michael Shearn, Axel Scherer, and Harry A. Atwater \\ Thomas J. Watson Laboratory of Applied Physics, California Institute of Technology, Pasadena, \\ California 91125, USA
}

(Received 9 October 2008; accepted 21 December 2008; published online 13 January 2009)

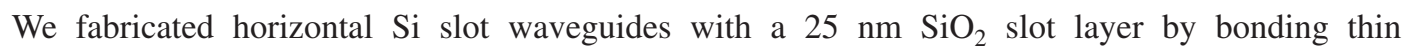
$\mathrm{Si}$-on-insulator wafers. After removing the $\mathrm{Si}$ substrate and buried oxide from one side of the bonded structure, grating-coupled waveguides and ring resonators were partially etched into the $\mathrm{Si} / \mathrm{SiO}_{2} / \mathrm{Si}$ device layers. The gratings exhibit efficiencies of up to $23 \%$ at $1550 \mathrm{~nm}$ and the ring resonators were measured to have loaded quality factors near 42000 for the lowest-order transverse-electric mode, corresponding to a propagation loss of $15 \mathrm{~dB} / \mathrm{cm}$. The leaky lowest-order transverse-magnetic mode was also observed with a propagation loss of $44 \mathrm{~dB} / \mathrm{cm}$. (C) 2009 American Institute of Physics. [DOI: 10.1063/1.3070541]
\end{abstract}

Over the last few years, Si photonics technology has made possible the dense integration of passive waveguides and resonators. Research in Si photonics is now addressing the challenge of realizing chip-scale Si-based active devices. In particular, the concept of generating and amplifying light at telecommunications frequencies on a $\mathrm{Si}$ chip has been of great interest. One promising approach is to integrate active gain materials directly into a $\mathrm{Si}$ waveguiding layer. Using a slot waveguide with a Si/slot/Si structure, one can take advantage of symmetry to maximize overlap of guided modes with an active slot material and potentially employ the $\mathrm{Si}$ layers for electrical injection. In addition, for transversemagnetic (TM) polarized modes, the electric field is enhanced in the slot by a factor of $n_{\mathrm{Si}}^{2} / n_{\text {slot }}^{2}$, making low-index slot materials advantageous for high modal confinement. ${ }^{1}$ Thus, with an active slot layer, and in particular one based on a low-index matrix such as rare earth doped glass, Si-based slot structures that exhibit gain and even lasing may be possible.,

While vertical slot waveguides in Si-on-insulator (SOI) have been demonstrated, ${ }^{4,5}$ it is difficult to fill the highaspect ratio slot using conventional thin-film deposition processes. Thus, we believe that fabricating structures in a horizontal configuration is more adaptable to applications where a deposited active slot material is to be used. In this paper, we present a technique for fabricating Si-based horizontal slot waveguides that is compatible with deposited active materials but does not require subsequent deposition of lossy amorphous or polycrystalline Si layers. Our process uses room-temperature covalent wafer bonding to transfer the $\mathrm{Si}$ device layer of a SOI wafer onto a second SOI wafer with a thin $\mathrm{SiO}_{2}$ slot layer in between, as shown in Fig. 1. The resulting layered structure has single-crystalline Si layers both above and below the slot. Although the devices presented here are passive, an active silica-based slot layer could be deposited prior to bonding in order to fabricate a variety of active devices.

To fabricate our wafer-bonded slot waveguides, we began with commercially available SOI wafers from SOITEC with a $3 \mu \mathrm{m}$ buried oxide layer and a $250 \mathrm{~nm}$ thick, lightly doped $\left(\sim 10^{15} \mathrm{~cm}^{-3}\right)$, $p$-type Si device layer that was thinned

\footnotetext{
a) Author to whom correspondence should be addressed. Electronic mail: rbriggs@caltech.edu.
}

to approximately $140 \mathrm{~nm}$ using wet thermal oxidation and buffered hydrofluoric acid (HF) etching. The wafers have a manufacturer-specified $3 \sigma$ thickness variation of $20 \mathrm{~nm}$ for the Si device layer; however, we measured variations of less than $5 \mathrm{~nm}$ over relevant device areas. A thin thermal oxide bonding layer was grown using dry oxidation. Cleaved pieces of the wafers were then prepared and bonded using a process from Tong et al. ${ }^{6}$ Specifically, the pieces were cleaned in a modified RCA solution of 1:0.2:5 $\mathrm{H}_{2} \mathrm{O}_{2}: \mathrm{NH}_{4} \mathrm{OH}: \mathrm{H}_{2} \mathrm{O}$, rinsed in de-ionized water, and dipped in 1:200 $\mathrm{HF}: \mathrm{H}_{2} \mathrm{O}$ for $10 \mathrm{~s}$. The samples were then baked at $250{ }^{\circ} \mathrm{C}$, cleaned again in the RCA solution, and treated in an $\mathrm{O}_{2}$ plasma at $400 \mathrm{~W}$ with an $\mathrm{O}_{2}$ pressure of 115 mTorr. After being placed in contact and left at room temperature for at least $24 \mathrm{~h}$, the samples were annealed at $1100{ }^{\circ} \mathrm{C}$ in $\mathrm{N}_{2}$.

The Si substrate on one side of the bonded structure was removed using a combination of mechanical lapping and

(a)

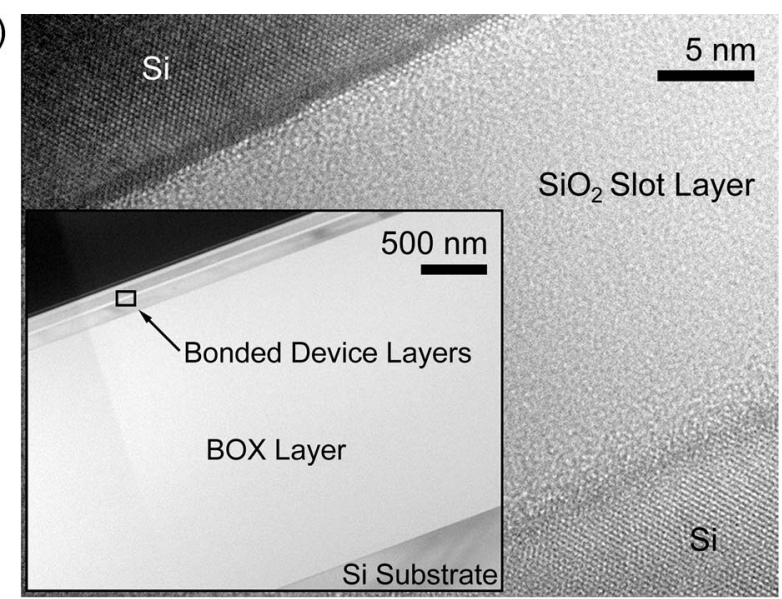

(b)

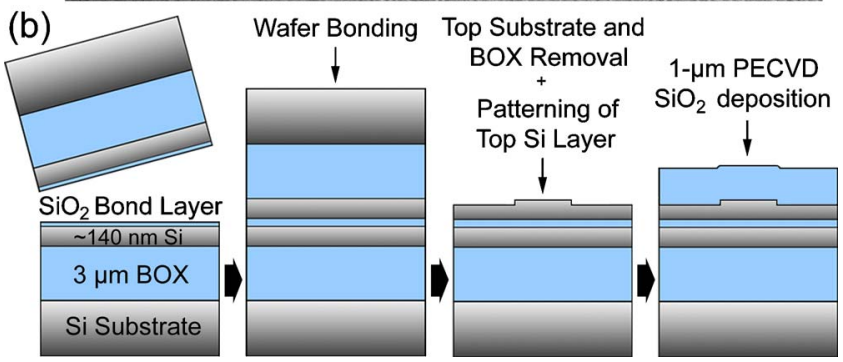

FIG. 1. (Color online) (a) Cross-sectional TEM micrographs of the fabricated structure, showing all layers (inset) and a higher magnification image of the bonded slot region. (b) A schematic of the fabrication process flow. 


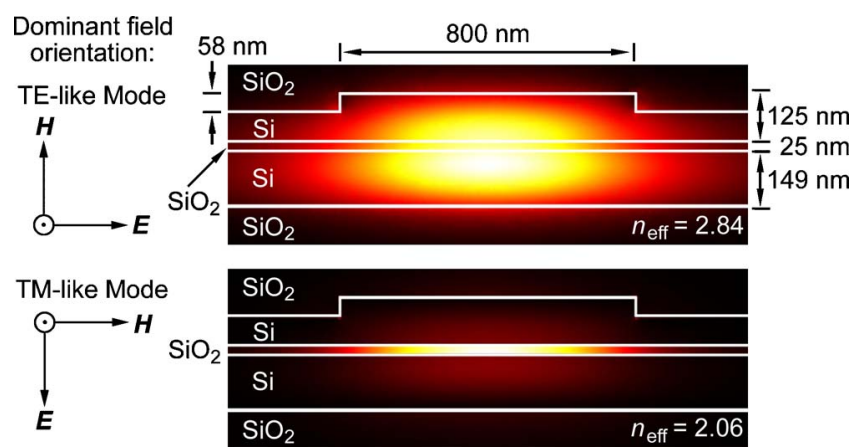

FIG. 2. (Color online) The as-fabricated waveguide geometry shown with the calculated power distribution in the guided TE-like and TM-like modes at $1550 \mathrm{~nm}$.

etching in a $20 \% \mathrm{KOH}$ solution. A lamella from the sample was extracted by focused-ion beam milling and characterized with high-resolution transmission electron microscopy (TEM), as shown in Fig. 1(a). The observed layer dimensions agree with those determined by spectroscopic ellipsometry (given in Fig. 2) within $\pm 2 \mathrm{~nm}$, and the Si layers are clearly single crystalline, as indicated by the appearance of lattice fringes in TEM.

Ring resonators with grating-coupled through and drop ports were fabricated using electron-beam lithography and a $\mathrm{SF}_{6} / \mathrm{C}_{4} \mathrm{~F}_{8}$ reactive-ion etching process. The gratings were designed for a depth of $50 \mathrm{~nm}$ in order to match their characteristic coupling length to the focused spot size of the laser beam used for device testing, ${ }^{7}$ and the actual etch depth was determined to be $58 \mathrm{~nm}$ using ellipsometry. A waveguide width of $800 \mathrm{~nm}$ was chosen in order to ensure that only the lowest-order transverse-electric (TE) and TM modes would be supported. The final cross-sectional waveguide geometry is shown in Fig. 2. The resonator device layout is shown in Fig. 3 along with scanning-electron microscopy (SEM) images of the etched structures. The gratings are $30 \mu \mathrm{m}$ wide and $50 \mu \mathrm{m}$ long with a pitch of $660 \mathrm{~nm}$. $500 \mu \mathrm{m}$ long linear transitions were pattered between the gratings and the narrower waveguides. Rings were patterned with a large inner diameter of $400 \mu \mathrm{m}$ to avoid bending loss, ${ }^{8}$ and $1 \mu \mathrm{m}$ coupling gaps were defined between the ports and the rings. Finally, the entire structure was coated with $1 \mu \mathrm{m}$ of $\mathrm{SiO}_{2}$ using plasma-enhanced chemical vapor deposition.

Light near $1550 \mathrm{~nm}$ was coupled from a New Focus 6428 Vidia Swept tunable diode laser into TE and TM modes using a lensed fiber focuser and a linear wavelength sweep rate of $1 \mathrm{~nm} / \mathrm{s}$. The focuser has an estimated working distance of $12.4 \mathrm{~mm}$ and a spot size of $8 \mu \mathrm{m}$. By varying the angle of the focuser relative to the grating, modes were accessed selectively according to their unique modal group velocities. A polarization controller was used to maximize the coupled intensity for each mode. Light was coupled out through an identical lensed fiber and into a high-speed InGaAs photoreceiver. Using the method of Striefer et al. ${ }^{9}$ the ideal coupling angle was calculated to be near $30^{\circ}$ offnormal for $\mathrm{TE}$ modes and $-30^{\circ}$ for $\mathrm{TM}$ modes [refer to the inset in Fig. 3(a)]. The ideal coupling angles were found experimentally to be $28.5^{\circ}$ and $-27^{\circ}$, respectively. By comparing the off-resonance through-port power transmission for a coupling angle of $28.5^{\circ}$ with the maximum possible transmitted laser power through our measurement system, we measured an overall attenuation of $12.6 \mathrm{~dB}$ through the de-

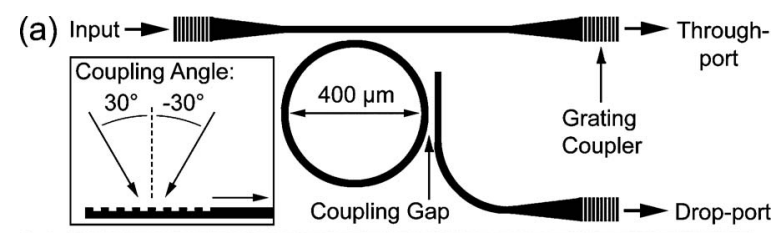

(b)

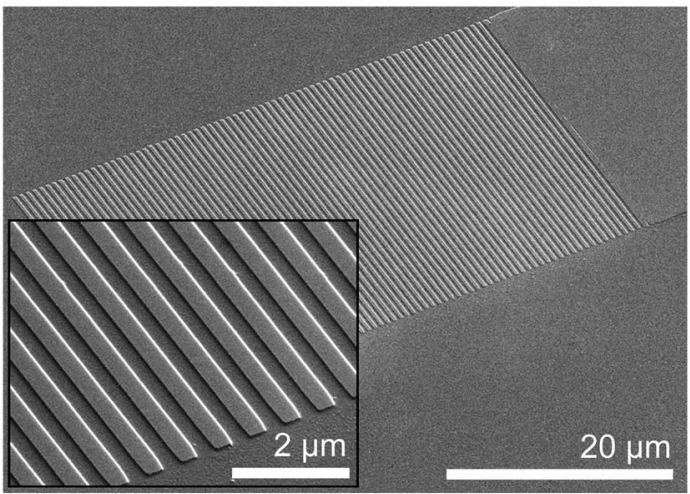

(c)

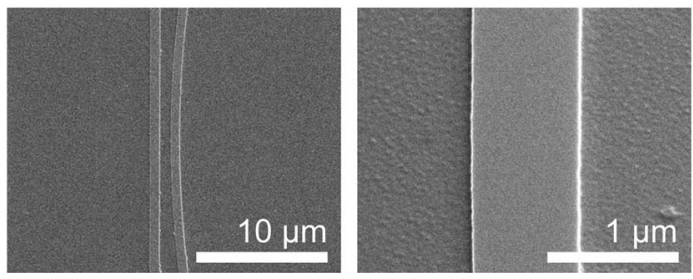

FIG. 3. (a) The ring resonator device layout and SEM images of (b) a grating coupler and (c) the coupling region between a drop port and a ring (with a higher magnification image of the ring waveguide).

vice. This corresponds to a $3.5 \mathrm{~dB}$ loss per coupler, or a grating-plus-taper coupling efficiency of $23 \%$ for the TE mode. The grating-plus-taper coupling efficiency for the TM mode was measured to be $7 \%$ for reasons discussed later.

Figure 4 shows the through- and drop-port response near

(a)

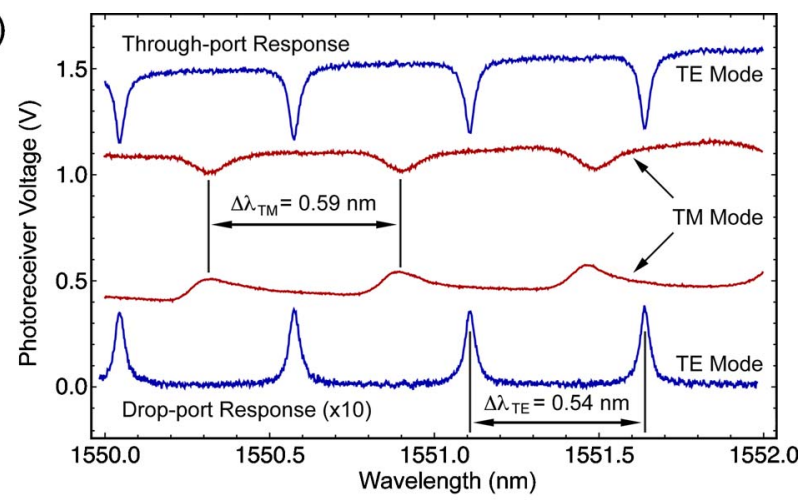

(b)

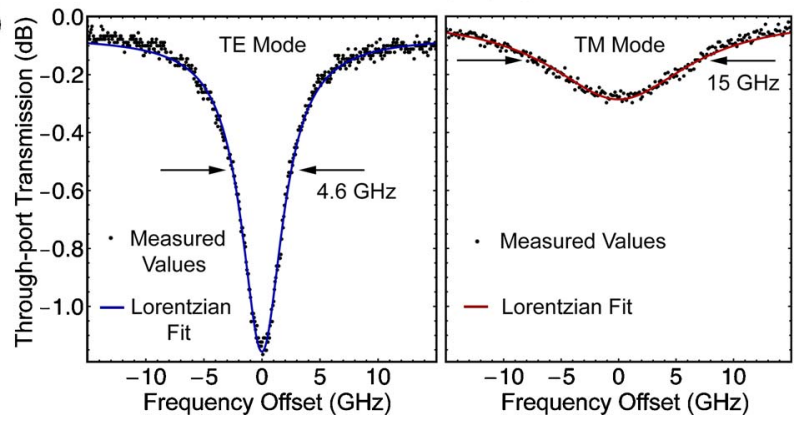

FIG. 4. (Color online) (a) Through- and drop-port response of a single ring resonator at both the TE and TM coupling angles. The TM mode response curves are offset for clarity. (b) Lorentzian fits of a through-port resonance for each mode. 
$1550 \mathrm{~nm}$ for a single device at both the TE- and TM-mode coupling angles. The modes can be distinguished by their characteristic free-spectral range (FSR), which is given in terms of wavelength as ${ }^{5}$

$$
\Delta \lambda=\lambda_{0}\left(1+n_{g} \frac{L}{\lambda_{0}}\right)^{-1}
$$

where $\lambda_{0}$ is the resonant wavelength, $L$ is the round-trip length of the cavity, and $n_{g}$ is the group index, defined in terms of the effective index, $n_{\text {eff }}$, as

$$
n_{g}=n_{\mathrm{eff}}-\lambda_{0}\left(\frac{\partial n_{\mathrm{eff}}}{\partial \lambda}\right)_{\lambda_{0}} .
$$

A finite-element mode solver (COMSOL) was used to determine $n_{\text {eff }}$ and its derivative near $1550 \mathrm{~nm}$ for each mode. It was found that $n_{g, \mathrm{TE}}=3.46$ and $n_{g, \mathrm{TM}}=3.22$, which predicts $\Delta \lambda_{\mathrm{TE}}=0.551 \mathrm{~nm}$ and $\Delta \lambda_{\mathrm{TM}}=0.592 \mathrm{~nm}$. For both polarizations, the FSR is slightly overestimated compared to the experimental values shown in Fig. 4(a), likely a result of variations in the geometry of the fabricated structure compared with the dimensions used in the calculations. In particular, $n_{\text {eff }}$ is fairly sensitive to the layer thickness and the etch depth.

By fitting Lorentzian functions to the through-port resonances, as shown in Fig. 4(b), the on-resonance extinction was determined to be $1.2 \mathrm{~dB}$ for the TE mode and $0.3 \mathrm{~dB}$ for the TM mode, indicating that the resonators are undercoupled and that the loaded $Q$ factor is a fair estimate of the intrinsic $Q$. As a result of the very low coupling efficiency of the TM mode, higher gain had to be used on the photoreceiver to resolve the TM drop-port resonances, resulting in an asymmetric lineshape. Based on the though-port response, the linewidths of the resonances are $4.6 \mathrm{GHz}$ for the TE mode and $15 \mathrm{GHz}$ for the TM mode, corresponding to $Q$ factors of 42000 and 13000 , respectively. The propagation loss (in decibel) is defined as $\alpha=4.343 \times 2 \pi n_{g} /\left(\lambda_{0} Q\right)$, so $\alpha_{\mathrm{TE}}=15 \mathrm{~dB} / \mathrm{cm}$ and $\alpha_{\mathrm{TM}}=44 \mathrm{~dB} / \mathrm{cm}$. The TE-mode loss, while high compared with partially etched nonslot SOI waveguides, ${ }^{8,10}$ is likely due in part to the relatively large sidewall depth and residual surface roughness from the partial Si etch, as seen in Fig. 3(c). The loss is comparable to previously reported narrow $(<1 \mu \mathrm{m})$ rib waveguides that have not undergone additional smoothing, ${ }^{11,12}$ and is an order of magnitude lower than slot waveguides with sputtered $\mathrm{Si}$ layers. $^{3}$

The TM waveguide mode has an effective index of $n_{\text {eff,TM }}=2.06$, while the TE slab mode in the partially etched regions has an effective index of $n_{\text {eff,TE }}^{\text {slab }}=2.68$. Thus, the guided TM mode can leak into the etched slab. It has been shown by Webster et al. ${ }^{13}$ for single-layer Si waveguides that there exist certain "magic" widths for which the TM mode has loss comparable to the TE waveguide mode. These widths are given by

$$
W_{m}=\frac{m \lambda_{0}}{\sqrt{\left(n_{\mathrm{eff}, \mathrm{TE}}^{\text {slab }}\right)^{2}-\left(n_{\mathrm{eff}, \mathrm{TM}}\right)^{2}}}, \quad m=1,2,3, \ldots
$$

For our geometry, $W_{1} \approx 900 \mathrm{~nm}$ when $\lambda_{0}=1550 \mathrm{~nm}$, significantly wider than our $800 \mathrm{~nm}$ waveguides. However, higherorder modes are supported if the waveguide ridge is made much broader than the width used in our design. To overcome this, the etch depth can be made shallower, at the expense of rendering the gratings less effective. Alternatively, a two-step etch process can be used, where the gratings are etched to the ideal depth while the waveguides are formed with either an extremely shallow etch, ${ }^{8,13}$ which would result in a smaller $W_{1}$, or an etch deep enough to cut off the TE mode in the slab. Here we point out that the lower gratingplus-taper efficiency mentioned previously for TM modes is due to leakage from the linear transitions, an issue that can be remedied with a deeper etch.

In conclusion, we demonstrated wafer-bonded horizontal slot waveguides with single-crystalline $\mathrm{Si}$ waveguiding layers. By coupling $1550 \mathrm{~nm}$ light into partially etched waveguide ring resonators using surface relief gratings, we were able to access the lowest-order TE and TM modes of the slot structure and determine the waveguide propagation loss. When compared with narrow single-layer SOI waveguides fabricated by a similar process, the measured TE-mode loss of $15 \mathrm{~dB} / \mathrm{cm}$ suggests that the bonded interface in the slot does not lead to overwhelming attenuation near $1550 \mathrm{~nm}$. The higher loss of $44 \mathrm{~dB} / \mathrm{cm}$ for the TM mode is due to leakage into the slab surrounding the waveguide and should be addressed in future devices by modifying the device geometry. Overall, we believe these results suggest that wafer bonding is a viable method for integrating a variety of silicabased slot materials directly into SOI waveguides.

The authors gratefully acknowledge H. J. Lezec and M. J. Archer for TEM assistance. R.M.B. also acknowledges the support of the NDSEG Fellowship and M.S. thanks the NSF for his Graduate Research Fellowship. This project was funded by the AFOSR under MURI Award No. FA9550-061-0470 as well as by the Kavli Nanoscience Institute and DARPA under EPIC Program No. HR0011-04-10054, and it made use of the facilities of the Center for Science and Engineering of Materials, an NSF MRSEC.

${ }^{1}$ V. R. Almeida, Q. Xu, C. A. Barrios, and M. Lipson, Opt. Lett. 29, 1209 (2004)

${ }^{2}$ C. A. Barrios and M. Lipson, Opt. Express 13, 10092 (2005).

${ }^{3}$ M. Galli, D. Gerace, A. Politi, M. Liscidini, M. Patrini, L. C. Andreani, A. Canino, M. Miritello, R. Lo Savio, A. Irrera, and F. Priolo, Appl. Phys. Lett. 89, 241114 (2006).

${ }^{4}$ Q. Xu, V. R. Almeida, R. R. Panepucci, and M. Lipson, Opt. Lett. 29, 1626 (2004)

${ }^{5}$ T. Baehr-Jones, M. Hochberg, C. Walker, and A. Scherer, Appl. Phys. Lett. 86, 081101 (2005).

${ }^{6}$ Q.-Y. Tong, G. Fountain, and P. Enquist, Appl. Phys. Lett. 89, 042110 (2006).

${ }^{7}$ D. Pascal, R. Orobtchouk, A. Layadi, A. Koster, and S. Laval, Appl. Opt. 36, 2443 (1997).

${ }^{8}$ M. A. Webster, R. M. Pafchek, G. Sukumaran, and T. L. Koch, Appl. Phys. Lett. 87, 231108 (2005).

${ }^{9}$ W. Streifer, D. R. Scifres, and R. D. Burnham, IEEE J. Quantum Electron. 12, 422 (1976).

${ }^{10}$ S. Lardenois, D. Pascal, L. Vivien, E. Cassan, S. Laval, R. Orobtchouk, M. Heitzmann, N. Bouzaida, and L. Mollard, Opt. Lett. 28, 1150 (2003).

${ }^{11}$ K. K. Lee, D. R. Lim, L. C. Kimerling, J. Shin, and F. Cerrina, Opt. Lett. 26, 1888 (2001).

${ }^{12}$ Y. A. Vlasov and S. J. McNab, Opt. Express 12, 1622 (2004).

${ }^{13}$ M. A. Webster, R. M. Pafchek, A. Mitchell, and T. L. Koch, IEEE Photon. Technol. Lett. 19, 429 (2007). 